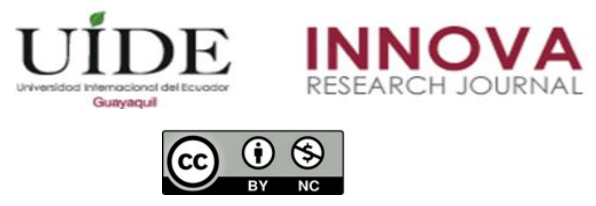

INNOVA Research Journal, ISSN 2477-9024

(Septiembre-Diciembre 2019). Vol. 4, No.3.2 pp. 98-113

DOI: https://doi.org/10.33890/innova.v4.n3.2.2019.1067

URL: http://revistas.uide.edu.ec/index.php/innova/index

Correo: innova@uide.edu.ec

\title{
La Andragogía, una estrategia de capacitación turística para el desarrollo sostenible del Chocó Andino de Pichincha
}

\section{The Andragogy, a touristic training strategy for the sustainable development of the Chocó Andino of Pichincha}

\author{
Pamela Caiza Vivas \\ Facultad Latinoamericana de Ciencias Sociales - FLACSO, Ecuador \\ Ximena Aguirre Ulloa \\ Jonathan Cruz Pierard \\ Universidad UTE, Ecuador
}

Autor por correspondencia: pcaizafl@flacso.edu.ec, pamela.caiza@ gmail.com; xaguirre@ute.edu.ec; jonathan.cruz@ute.edu.ec

Fecha de recepción: 12 de julio de 2019 - Fecha de aceptación: 20 de noviembre de 2019

\begin{abstract}
Resumen
La capacitación para personas que desarrollan sus actividades productivas en ecosistemas considerados como vulnerables tienen como eje fundamental la conservación de la riqueza biológica y cultural; este proceso evidencia como problemática la falta de metodologías específicas que se adapten a las realidades socioeconómicas, ambientales y geográficas de las zonas. La investigación tuvo como objetivo crear una metodología que se convierta en una verdadera herramienta de capacitación para pequeños emprendedores turísticos en el Chocó Andino de Pichincha. La investigación se diseñó de tipo cualitativo - exploratorio, siendo el principal método de investigación el estudio de caso, a la par se plantearon como herramientas o técnicas a la revisión bibliográfica, y un acercamiento etnográfico a través de entrevistas semiestructuradas y grupos focales con enfoque acción - participación. El estudio permitió determinar: actores, involucrados y necesidades de capacitación; logrando concluir que el modelo andragógico es el método que se adapta a la realidad demográfica de la zona, al favorecer contextos basados en el diálogo, y participación. La propuesta de Metodología Andragógica desarrolló un criterio de compromiso entre los participantes, y constituye una herramienta de capacitación para pequeños emprendedores turísticos que puede adaptarse a zonas geográficas similares o con características socioeconómicas semejantes.
\end{abstract}

Palabras claves: capacitación turística; conservación; emprendedores turísticos; desarrollo sostenible; andragogía

\begin{abstract}
The training for people who develop their productive activities in ecosystems considered as vulnerable, has as a fundamental axis the conservation of the biological and cultural richness; this process shows as problematic the lack of specific methodologies that adapt to the socioeconomic and environmental realities of these zones. This investigation had as principal aim to create a
\end{abstract}


training's methodology for small touristic entrepreneurs of the Choco Andino Region of Pichincha, through the analysis of the association's members and the entrepreneurship's characteristics, to improve the quality of the touristic services. To reach these goals, a qualitative, descriptive and exploratory research was performed, the most reliable form of research was the case study with tools such as: bibliographic review, and an ethnographic approach with semi-structured interviews and focus groups discussions. The study allows to determine: actors and needs of training, concluding that the andragogic model is the best training tool, because it promotes contexts based on frank dialogue and wide participation. The Andragogic Methodology achieved a consensus level among all participants, it is based on the labor competences model and it constitutes a training tool for small touristic entrepreneurs that can be adapted to areas with similar geographical and socioeconomic characteristics.

Key words: touristic training; conservation; touristic entrepreneurs; sustainable development; andragogy

\section{Introducción}

\section{Problemática}

La educación de las personas en ecosistemas considerados como vulnerables, requiere de una visión a largo plazo del capital humano, por lo que es importante desarrollar un enfoque que permita orientar las acciones en el tiempo, a través de la consolidación de grupos de trabajo con capacidades para enfrentar los distintos desafíos que implica el proceso de construcción de destinos turísticos sustentables.

En este marco interesan dos puntos: a) aprovechar y direccionar todo el conocimiento local para consolidar procesos metodológicos de enseñanza que van más allá de la simple escolarización; y b) construir un esquema de capacitación integral donde se consolide lo ambiental, turístico y empresarial. Se deben crear sinergias de conjunto entre los actores sociales de un destino turístico, con el objetivo de fomentar un capital social genuino, donde "las personas, las familias, los grupos son portadores de actitudes de cooperación, valores, tradiciones, visiones de la realidad, que son su identidad misma" (Otero, 2006, p. 66).

Buscar el crecimiento económico, turístico y ambiental de la ecoregión del Chocó Andino de Pichincha implica establecer procesos de capacitación adaptados a la realidad socioeconómica y ambiental de la zona, formar gestores de turismo capaces de identificar fortalezas, oportunidades, debilidades y amenazas con una comprensión holística de las relaciones sociales existentes. Entonces ¿cómo establecer una metodología de capacitación enfocada para el sector turístico en el Chocó Andino de Pichincha que atienda a la realidad socioeconómica - ambiental?

\section{Justificación}

Se debe partir del capital social del destino turístico Chocó Andino para evaluar las alternativas de capacitación, y que constituya el umbral base sobre el cual se debe trabajar comunitariamente la metodología, participación y compromiso de los emprendedores turísticos. "El control local es un componente indispensable para mantener una relación de equilibrio entre la conservación y el turismo" (Otero, 2006, p. 69), partiendo de esta afirmación es que el manejo 
de la actividad turística ambiental debe estar asociada a las prácticas socioculturales de la zona, y estas a su vez estar potenciadas en la formación de emprendedores a escala local con intervención del sector público, privado y la academia.

La metodología pretende orientar a todos los actores locales de la zona ver en la actividad económica turística una opción de desarrollo sostenible en la que interactúen la conservación de la biodiversidad, la generación de recursos económicos y el mejoramiento de las condiciones sociales de la población. Se debe partir del capital social del destino turístico Chocó Andino de Pichincha para evaluar las alternativas de capacitación, y que constituya el umbral base sobre el cual se debe trabajar comunitariamente la metodología, participación y compromiso de los emprendedores turísticos. "El control local es un componente indispensable para mantener una relación de equilibrio entre la conservación y el turismo" (Otero, 2006, p. 69), partiendo de esta afirmación es que el manejo de la actividad turística ambiental debe estar asociada a las prácticas socioculturales de la zona, y estas a su vez estar potenciadas en la formación de emprendedores a escala local con intervención del sector público, privado y la academia.

\section{Contexto Geográfico}

La ecorregión del Chocó Andino de Pichincha es considerado como un territorio particular y susceptible debido a características socioculturales, ambientales y económicas, razones por las que amerita ser nombrada como una micro-región dentro de la provincia. Desde tiempos históricos, el área fue un sitio de transición entre las regiones Costa y Sierra, uno de los grupos humanos más significativos fue el pueblo de los Yumbos. El aspecto ambiental es sin duda alguna, uno de los puntos más importantes de la zona, característica relevante es la gradiente altitudinal que va desde los $400 \mathrm{msnm}$ hasta los $4780 \mathrm{msnm}$, formando diferentes zonas de vida y formaciones vegetales; razones por las cuales la región pertenece al hotspot ${ }^{1}$ Tumbés-Chocó Magdalena, identificada como una de las zonas más importantes del mundo para la conservación, debido a la gran diversidad biológica (227 especies de orquídeas, más de 600 especies de aves de las cuales 80 son endémicas) y a las fuertes presiones que enfrenta. Razones por las cuales en julio 2018, 286000 hectáreas de la zona noroccidental de Pichincha fueron declaradas como Reserva de la Biósfera Chocó Andino, que incluye nueve bosques protectores, tres Áreas de Conservación y Uso Sustentable - ACUS, cuatro Áreas de importancia para la conservación de aves - IBAS, múltiples reservas privadas, una reserva geobotánica, y ocho áreas núcleo $^{2}$ (Gobierno Provincial de Pichincha, 2015).

En el ámbito económico, las actividades agrícolas y ganaderas son las más generalizadas; sin embargo, los cambios actuales en la zona han ido incluyendo nuevas actividades en los habitantes del noroccidente de Pichincha, los modos de vida se han ido inclinando hacia actividades comunitarias sostenibles, incrementando los esfuerzos por conservar la biodiversidad, se nombran tres dinámicas históricas que han incidido y son: "el establecimiento de áreas protegidas por iniciativa privada; la consolidación de la región como destino turístico y,

\footnotetext{
${ }^{1}$ Punto caliente de biodiversidad. Son los lugares de la Tierra con mayor riqueza biológica y con un alto nivel de especies endémicas, denominadas así por ser exclusivas de determinadas regiones del planeta.

${ }^{2}$ Lugares donde los bosques tienen un mejor estado de conservación y son importantes por su biodiversidad y por los servicios ambientales que proveen.
}

Esta obra se comparte bajo la licencia Creative Common Atribución-No Comercial 4.0 International (CC BY-NC 4.0) Revista de la Universidad Internacional del Ecuador. URL: https://www.uide.edu.ec/ 
el desenvolvimiento de políticas estatales con respecto a la planificación y el ordenamiento territorial en el ámbito seccional" (Zalles, 2016, p. 68).

\section{Método}

El estudio de caso determina a la ecoregión del Chocó Andino de Pichincha como la unidad de análisis, al ser considerada como una zona donde se concilia la conservación de la biodiversidad y la actividad humana a través del uso sostenible de los recursos naturales. La investigación buscó determinar las necesidades de capacitación de los actores locales, por lo que es fundamental determinar metodologías de capacitación adaptadas a la realidad económica, ambiental y social de la comunidad. La revisión bibliográfica implicó el sustento de la línea base teórica para el análisis de la Andragogía; se desarrolló una investigación de campo mediante un acercamiento etnográfico, donde se llevaron a cabo herramientas de tipo cualitativo. Las entrevistas semiestructuradas, para determinar los actores principales, involucrados y la trayectoria del turismo en la zona. La observación participativa, mediante el involucramiento con los Grupos de Apoyo Local - GAL ${ }^{3}$, permitió observar y participar de la dinámica de aviturismo logrando identificar: fortalezas, oportunidades, debilidades y amenazas. Grupos focales con los miembros de los GAL, cuya información será relevante al momento de triangular los datos para identificar las necesidades de capacitación de tipo urgente.

\section{Marco Teórico}

\section{Andragogía}

Para hablar de Andragogía, de educación de adultos, se debe empezar por entender el contexto de educación como una ciencia para diferenciar términos que si bien están relacionados con la educación, al profundizar resultan completamente diferentes. Entonces, se comprende a la Educación (Carr, 2005) como aquel proceso de socialización por medio del cual una sociedad transmite formalmente a los nuevos miembros, una serie de conocimientos, valores, lineamientos, procedimientos y directrices como normas e instrumentos de desempeño en los diferentes ámbitos de la vida de un individuo.

La educación y la capacitación como un proceso educativo, según Shalk Quintana (2009), es un proceso sociocultural permanente, intencionado y sistemático dirigido al perfeccionamiento y realización del ser humano como persona y al mejoramiento de las condiciones que beneficien el desarrollo y transformación de la calidad de vida. El ser que se capacita realiza una interacción consigo mismo (autoestructuración) y con su mundo laboral (heteroestructuración) mediante la elaboración cognitiva y reasunción afectiva, cuyo resultado es el ejercicio de la autonomía, su responsabilidad y su compromiso con el entorno donde se desarrolla.

La educación para adultos según Requejo (2003), busca garantizar que haya altos niveles de participación adulta en las actividades de educación - formación, y al mismo tiempo busca

\footnotetext{
${ }^{3}$ Grupo de actores locales dentro de las cuatro IBAs (Áreas de importancia para la conservación de aves) del Chocó Andino de Pichincha, y constituyen una base multiplicadora de acciones locales a favor de la conservación de los recursos y biodiversidad.
} 
garantizar la igualdad entre los individuos y los subgrupos de la población, realizando programas de calidad con los recursos necesarios. Es importante invertir en la educación para adultos ya que contribuye a aumentar el capital humano de los individuos y los convierte en personas más eficientes y capaces.

La inversión en el recurso humano ayudará a lograr un equilibrio entre las necesidades de todos los actores sociales. En la medida en la que el ser humano avanza en su crecimiento, es muy claro que el tipo de relación educativa y de aprendizaje es y debe ser distinta. El estudio dirigido, planificado y evaluado que es propio de la Pedagogía, cambia en el modelo andragógico, ya que se impone el estudio autodirigido por decisiones participativas de los educandos. La Andragogía a diferencia de la Pedagogía centra su atención en el alumno y no en el profesor, "la adultez es asumida no como un problema cronológico sino como actitudinal, la adultez es la aceptación de la cultura previa del alumno, de su capacidad de generar conocimiento, y de reconocer sus necesidades y expectativas” (Rodríguez Rojas, 2003, p. 84).

El sistema de ideas de Dewey (1938) es el que ha tenido más influencia sobre la enseñanza de adultos, ya que contrasta los principios básicos de la educación tradicional con los de la enseñanza eficaz:

A la disciplina tradicional se le opone la actividad libre, al aprendizaje mediante textos y profesor se le opone el aprendizaje mediante la experiencia; a la adquisición de habilidades aisladas y técnicas metódicas de enseñanza se le opone su adquisición de significado como medios para obtener los fines que dirigen hacia un interés vital (Dewey, 1938, p. 5-6).

El sistema de Dewey gira alrededor de algunos conceptos clave tales como: toda educación genuina viene de la experiencia, la democracia para promocer calidad en la experiencia humana, la continuidad ya que cada experiencia toma algo de la anterior y de alguna manera modifica la realidad de las posteriores, la interacción donde se asigna valores iguales a la experiencia y a la fuerza educativa.

El rol del participante adulto, según (Benne, 2005) en el proceso de aprendizaje, es diferente y se proyecta con un mayor alcance que el de ser un receptor pasivo, tomador de apuntes, conformista, resignado memorista o simple repetidor de las enseñanzas impartidas por un instructor, docente o facilitador; la participación implica el análisis crítico de las situaciones planteadas, a través del aporte de soluciones efectivas. La Andragogía también conocida como la disciplina propia y específica para el estudio e investigación de la Educación para adultos, no únicamente pretende ser un método educativo enfocado en la gente adulta, sino que se conecta con la educación permanente evitando la discontinuidad con otras etapas de la vida humana, satisface la necesidad de una educación coextensiva a la vida misma en el sentido que se trata de "volver a aprender" o en términos actuales "aprender a aprender". (Requejo, 2003, p. 9) sostiene que "La idea de educación permanente no pretende crear un sistema paralelo al sistema escolar o universitario sino englobar todas las formas de educación, la totalidad de la población y las diferentes edades de la vida". 
Los principios psicopedagógicos operativos, son aquellos que permiten la acción, es decir como se va a lograr la educación de adultos, según (Ludojoski, 1978, p. 80) menciona que son los siguientes:

- Empleo de ejemplos clasificadores, tomados de la experiencia.

- Necesidad de facilitar la comprensión experimental de los contenidos de la enseñanza.

- La búsqueda, de la verdad, como tarea exclusiva del discente (alumno, el que aprende)

- No, al enciclopedismo escolar. Si, a la investigación personal del discente.

- El tema de la lección, en conexión con alguna situación de vida cercana al discente.

- La situación didáctica, un alumno que pregunta y un maestro que responde.

- Buen maestro no es quien mucho enseña, sino quien enseña a pensar personalmente al alumno.

- Necesaria síntesis didáctica entre los contenidos a aprender y el razonamiento personal del discente.

- Pensar o elaborar juicios personales sobre un tema a estudiar.

- Tener en cuenta las características de las funciones cognoscitivas psicológicas

La Andragogía basa su praxis en el principio de horizontalidad, "donde el adulto aprende lo que quiere y cuando lo quiere hacer" (Torres, Fermín, Arroyo, \& Piñero, 2000, p. 26). La educación para adultos (Requejo, 2003) busca garantizar que haya altos niveles de participación adulta en las actividades de educación y formación y al mismo tiempo busca garantizar la igualdad entre lo individuos y los subgrupos de población, realizando programas de calidad que cuenten con los recursos necesarios. Es importante invertir en la educación para adultos ya que la educación y formación contribuyen a aumentar el capital humano de los individuos y los convierte en trabajadores más eficientes, en verdaderos ciudadanos para la sociedad que demanda día a día gente mucho más preparada.

\section{Capacitación}

Desde el momento que se propuso la Calidad como modelo de gestión a los en la década de los 50, el papel de los trabajadores en las organizaciones productivas, cambió radicalmente. A partir del esquema de la reacción en cadena que provoca la calidad, propuesta por Deming (1989) hacia la competitividad de la empresa, donde se afirma que la calidad conduce inicialmente a la productividad, porque se cometen menos errores, surge la necesidad de capacitar individuos para que realicen sus actividades. (Pérez, Pineda, \& Arango, 2011, p. 10). La cultura de cualquier tipo de organización se enriquece a través de la administración participativa que a la vez puede conducir al desarrollo de las habilidades y destrezas de todos los actores involucrados. Por ejemplo, (Nonaka, Toyoma, \& Konno, 2000) plantean que si se crean las condiciones para la interacción y participación en los lugares de trabajo, lo que es fundamental para el aprendizaje, finalmente éste impactará en el aumento de la productividad.

La capacitación es el desarrollo de habilidades específicas para un determinado puesto de trabajo, los procesos de capacitación permiten el desarrollo de competencias asociadas, como lo propone McClelland (1973) quien estableció dos métodos fundamentales que facilitan su enfoque: 
- Muestras de Desempeño (personas con desempeño excelente vs personas con desempeño promedio).

- Entrevistas de Eventos Conductuales. McClelland (1973) confirmó que los indicadores tradicionales de desempeño, tales como pruebas de aptitud, resultados de exámenes y referencias, no predecían realmente el desempeño en un puesto.

El principio básico del "enfoque de competencias conductuales es que lo que las personas piensan o dicen acerca de sus motivos o habilidades no es creíble, solamente lo es, aquello que han hecho efectivamente en los eventos más críticos que han enfrentado" (Pérez, Pineda, \& Arango, 2011, p. 12). La creación de las condiciones necesarias para generar procesos de aprendizaje en cualquier tipo de organización, permite a todos los actores a asumir responsabilidades e iniciativas que impacten en el mejoramiento continuo de los procesos. Según Aguilar (2014, p. 43) la finalidad de la capacitación es "que la gente obtenga mayores conocimientos para que desarrolle su intelecto y así pueda tomar decisiones y desarrolle su papel como individuo dentro de la sociedad".

En el proceso de capacitación, "los instructores son los responsables de establecer un clima de de sinergia, de apertura y fundametalmente de participación para que la gente comparta sus experiencias y tenga la predisposición de escuchar a los demás" (Aguilar, 2014, p. 43). De esta forma se contribuye a procesos participativos donde todos los participantes encuentren un marco de referencia para discutir problemas, soluciones, experiencias. En la capacitación no se recurren a libros, por el contrario se utilizan manuales elaborados en base a la experiencia de los actores, conviertiéndose en productos didácticos a partir de los cuales se fomenta la discusión, intercambio de ideas para poder llegar a conclusiones colectivas; de esta forma la información que se recibe del entorno es interpretada, codificada y re-interpretada por la mente que va reconstruyendo y construyendo progresivamente modelos explicativos cada vez más complejos. El proceso de aprendizaje en la capacitación "debe ser breve, claro, conciso, de un alto impacto y debe garantizar resultados" (Aguilar, 2014, p. 44); de esta manera el conocimiento es el resultado de un proceso dinámico e interactivo.

El diseño curricular de la capacitación debe "procurar constituirse en uno de los medios que orienta la formación profesional, para lo cual se propone articular las características, las necesidades, y las perspectivas de la práctica profesional, con las del proceso formativo" (Catalano, Avolio de Cols, \& Sladogna, 2004, p. 76). El diseño metodológico y curricular de la capacitación "es un proceso dinámico donde la planificación se va ajustando, y retroalimentando de las experiencias que se presentan en la realidad social y sobre todo de diferentes problemas profesionales" (Aguilar, 2014, p. 36). Todo proceso de capacitación debe entenderse como un proceso de planificación pedagógica para la formación de técnicos superiores capaces de intervenir en la realidad social, a través de soluciones viables y pertinentes a problemas profesionales.

\section{Desarrollo Sostenible}

El concepto desarrollo sostenible "se relaciona directamente con la llamada crisis ambiental, que no es un fenómeno reciente... los problemas socioambientales generados por el neoliberalismo, como modelo de desarrollo depredador de la naturaleza y de las culturas, 
comienzan a evidenciarse precisamente en las últimas décadas del siglo XX” (Gallegos, 2009, $\mathrm{s} / \mathrm{p}$ ); determinando que el desarrollo sostenible busca mantener un equilibrio entre el crecimiento económico, ambiental y social al fomentando estas capacidades en el ser humano.

En 1972, el Club de Roma publica el estudio Los límites al crecimiento, que destaca tres principales conclusiones: a) si las tendencias de crecimiento, producción, contaminación y agotamiento de recursos, continúan los límites del crecimiento se alcanzarán antes de los 100 próximos años; b) indica la posibilidad de una estabilidad económica y ecológica sostenible en el futuro, a través del equilibrio de las necesidades materiales básicas de las personas y de las oportunidades que tengan de desarrollar su potencial humano individual; y c) mientras más pronto se tome la decisión de trabajar en este sentido, mayores son las posibilidades de lograr el éxito. (Meadows, 1983).

En 1972 la Conferencia de las Naciones Unidas sobre Medio Humano en Estocolmo ${ }^{4}$, "atenta a la necesidad de un criterio y unos principios comunes que ofrezcan a los pueblos del mundo inspiración y guía para preservar y mejorar el medio humano" (ONU, 1972, p. 3). Esta conferencia marca el inicio a nivel mundial para establecer límites a la racionalidad económica y delinear los desafíos que genera la degradación ambiental para la humanidad. En 1987, se publicó el informe "Nuestro Futuro Común" conocido como Informe de Brundtland, el informe se centra en "la crítica al modelo de desarrollo impuesto por los países del norte, enfatiza el desequilibrio entre los patrones de producción y consumo norte - sur” (Caiza, 2018, p. 3).

Existe una gran contradicción contenida en el concepto de desarrollo sostenible que se mantiene a lo largo del tiempo, y es que para alcanzar el tan anhelado desarrollo sostenible es necesario continuar impulsando el crecimiento económico de los sectores productivos del mundo. Para Leff (2000, p. 3) la sostenibilidad abre el debate sobre los fundamentos de la "economía, cuestionando el funcionamiento y cumplimiento de sus principios individualistas y mecanicistas: la utilidad marginal, la optimización en la asignación de recursos escasos y agotables a diferentes fines; el mercado como lugar donde los sujetos expresan sus preferencias individuales".

El tránsito hacia "la sostenibilidad implica que la producción, como base de la vida social, ha sido superada por la modernidad; entonces la globalización económica transforma al ambiente y aparecen las luchas sociales por la propiedad y el control de los recursos naturales" (Caiza, 2017, p. 2). La sostenibilidad sigue la estrategia teórica de basarse en "la economía para recodificar todas las formas de la naturaleza en términos de valores económicos, englobando a los bienes y servicios ambientales dentro del concepto totalizador de capital natural" (Leff, 2000, p. 3), es decir, abre un campo "hacia las interrelaciones con otros sistemas, en un afán de construir un paradigma sistémico-interdisciplinario-sostenible” (Leff, 2000, p. 3).

\footnotetext{
${ }^{4}$ La Conferencia de las Naciones Unidas sobre el Medio Humano se celebró en Estocolmo, en junio de 1972, contó con la participación de 1200 delegados de 110 países. Por vez primera, el eje ambiental se tomó en cuenta como elemento condicionador y limitante del modelo tradicional de crecimiento económico. Las deliberaciones se desarrollaron en tres ejes: a) necesidades sociales y culturales de planificación ambiental, b) recursos naturales, c) los medios para luchar contra la contaminación. Se aprobaron 26 principios y 103 recomendaciones.
} 
En palabras de Martínez Alier, la categoría sostenibilidad débil "permite la sustitución del capital natural por el capital hecho por los humanos. Lo que importa es que no disminuya el stock total de capital" (Martínez Alier y Roca Jusmet, 2013, p. 475). Entendiendo al capital natural como todos los activos naturales, o flujo de bienes y servicios útiles o renta natural; mientras que el capital hecho por los humanos es el capital fabricado, artificial o manufacturado. Entonces, toda sociedad que reduzca su capital natural y aumente su capital fabricado compensando esa pérdida para mantener el capital total, es una sociedad que alcanza la sustentabilidad débil.

La sostenibilidad fuerte en palabras de Gudynas "advierte que no toda la naturaleza puede ser reducida a un capital natural, ni que todas las valoraciones son económicas" (Gudynas 2009, 15). "Este enfoque no acepta la idea neoclásica de la plena sustituibilidad entre los diferentes tipos de capital" (Caiza y Peralta, 2017, p. 8), el capital natural no puede sustituirse por el capital manufacturado. "La sustituibilidad del capital natural está seriamente limitada por características ecológicas como la integridad, la irreversibilidad, la incertidumbre y la existencia de componentes críticos del capital natural indispensables para el bienestar humano" (Castiblanco Rozo, 2015, p. 4). Entonces, la sustituibilidad es un estado deseable para alcanzar la conservación del stock de capital natural por encima del mínimo deseado.

\section{Resultados}

En esta investigación se tomó el modelo de Requejo (2003) quien indica que el ciclo andragógico se estructura en cinco fases que definen un conocimiento y praxis educativa diferenciada:

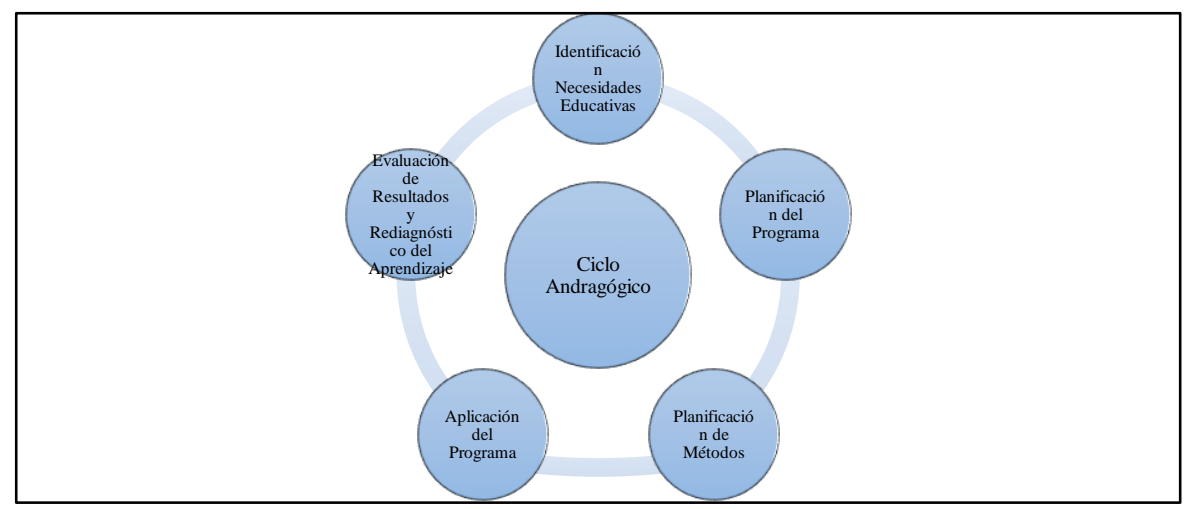

Figura 1 Ciclo Andragógico. Según Agustín Requejo, en el libro Educación Permanente y Educación de Adultos, 2003.

\section{Identificando necesidades}

Una vez aplicadas las herramientas de campo con los miembros de los GAL de las cuatro IBA's del Chocó Andino de Pichincha se determinaron necesidades de capacitación urgentes. Para el desarrollo de la metodología andragógica, se establecieron tres grandes ejes (ambiental, turístico y empresarial) y dentro de cada uno 26 módulos a ser impartidos, lo que permite desarrollar las destrezas y habilidades de los participantes al tener definida la línea base conceptual. La metodología busca desarrollar un criterio de compromiso y de competencias 
laborales, mediante la identificación de los siguientes ejes transversales: a) concientizar y entender el papel que juega cada individuo en relación al medio ambiente y su impacto como agente contaminante, $\mathrm{b}$ ) establecer y crear un real compromiso con el medio ambiente, $\mathrm{c}$ ) implementar prácticas empresariales amigables con el medio ambiente en las actividades productivas.

\section{Tabla 1}

Ejes Temáticos de la Metodología Andragógica para pequeños emprendedores del Chocó Andino de Pichincha

\begin{tabular}{|c|c|c|}
\hline Eje Ambiental & Eje Turístico & Eje Empresarial \\
\hline $\begin{array}{ll}\text { - } & \text { Biología General } \\
\text { - } & \text { Ecología General } \\
\text { - } & \text { Flora y Fauna Ecuatoriana } \\
\text { - } & \text { Introducción a la Ornitología } \\
\text { - } & \text { Aviturismo } \\
\text { - } & \text { Educación e Interpretación } \\
\text { - } & \text { Ambiental } \\
\text { Impactos Ambientales }\end{array}$ & $\begin{array}{ll}\text { - } & \text { Introducción al Turismo } \\
\text { - } & \text { Hospedería } \\
\text { - } & \text { Ama de Llaves } \\
\text { - } & \text { Geogrimentos y Bebía Turística } \\
\text { - } & \text { Técnicas de Guiar } \\
\text { - } & \text { Turismo de Aventura } \\
\text { - } & \text { Turismo Comunitario } \\
\text { - } & \text { Ecoturismo } \\
\text { - } & \text { Turismo Sostenible } \\
\text { - } & \text { Técnicas de manejo de } \\
& \text { Información Turística }\end{array}$ & $\begin{array}{ll}\text { - } & \text { Emprendimientos Turísticos } \\
\text { - } & \text { Negocios Productivos } \\
\text { - } & \text { Legislación Turística } \\
\text { - } & \text { Contabilidad Básica } \\
\text { - } & \text { Marketing Turístico } \\
\text { - } & \text { Técnicas de Comercialización } \\
\text { - } & \text { A Ventas } \\
\text { Atención al Cliente }\end{array}$ \\
\hline
\end{tabular}

\section{Planificando el Programa}

Una vez establecidas las necesidades de capacitación, se procedió a establecer el perfil del capacitado determinando dos figuras: a) Promotor Turístico Local Polivalente - PTLP aquellas personas con las siguientes características: proveedor de servicios turísticos, iniciador de propuestas, impulsador de iniciativas, emprendedor de un servicio y comunicador - difusor quienes requieren capacitación en los tres ejes temáticos; b) Informante Turístico Local - ITL personas que desarrollen un vínculo cercano con la actividad turística, son los encaminados a informar y orientar no solo a turistas sino también a la propia comunidad, este perfil no requiere de capacitación en el eje empresarial.

Para seleccionar a los participantes se creó un sistema de evaluación que permite determinar el nivel de instrucción de los aspirantes e identificar dos áreas de conocimientos: a) conocimientos generales y concordancias entre conceptos y objetos de estudio, b) relación causa - efecto para identificar la capacidad básica de relación visual. El puntaje ponderado para determinar el nivel de participación identificó que: a) se accede al nivel PTLP con un puntaje entre 7 y 10 puntos, y b) se accede al nivel ITL con un puntaje entre 4 y 6 puntos.

La metodología está basada en el desarrollo de competencias laborales en turismo, según Shalk Quintana (2009) la adquisición de paquetes de información y conocimiento fragmentado para la capacitación ya no es suficiente, ahora se requiere desempeños inteligentes que sepan hacer uso de los saberes construidos relacionándolos de distintas maneras para afrontar y resolver situaciones diversas en la vida productiva, social y personal. Establecer competencias en el ámbito turístico resulta complejo ya que implica aprender un saber, aprender a hacer y 
aprender a desarrollar actitudes específicas instrumentales, personales y sistémicas. Para los tres ejes metodológicos se desarrolló un esquema de capacitación, donde a cada asignatura se le asignó la carga horaria teórica y práctica de acuerdo a los contenidos a desarrollarse; al igual que los pre-requisitos para avanzar en el programa.

Planteado el esquema de capacitación para los emprendedores turísticos del Chocó Andino, se establecieron las guías metodológicas para las 26 asignaturas; los tres ejes de capacitación se deben aplicar bajo el modelo andragógico, con el fin que sean los propios participantes quienes realicen dinámicas, actividades y tareas para garantizar el aprendizaje. A manera de ejemplo para este artículo, se ha seleccionado una asignatura dentro de cada eje para que se visibilice el alcance metodológico de la propuesta.

Tabla 2

Ejemplos de guías metodológicas en los tres ejes

\begin{tabular}{|c|c|c|c|c|c|}
\hline \multicolumn{2}{|r|}{ Eje } & \multicolumn{2}{|r|}{ Eje Turístico } & \multicolumn{2}{|c|}{ Eje Empresarial } \\
\hline \multirow{9}{*}{$\begin{array}{l}\text { Modulo I } \\
\text { Definició } \\
\text { n y } \\
\text { Caracterí } \\
\text { sticas }\end{array}$} & Ecología General & \multicolumn{2}{|c|}{ Introducción al Turismo } & \multicolumn{2}{|c|}{ Negocios Productivos } \\
\hline & Relación y diferencias entre & \multirow{8}{*}{$\begin{array}{l}\text { Módulo } \\
\text { I } \\
\text { Anteced } \\
\text { entes del } \\
\text { Turismo }\end{array}$} & Definiciones & \multirow{7}{*}{$\begin{array}{l}\text { Módulo } \\
\text { I Bases } \\
\text { Concept } \\
\text { uales }\end{array}$} & Definición de \\
\hline & Biología y Ecología & & Básicas & & Negocio \\
\hline & Definición de Ecología e & & Superestructura & & Niveles de \\
\hline & Importancia & & del Turismo & & Compromiso \\
\hline & Definición de Ecosistema & & Elementos del & & Tipos de Negocios \\
\hline & & & Turismo & & Productivos \\
\hline & Componentes de un Ecosistema & & Atractivos & & Uso de recursos \\
\hline & $\begin{array}{l}\text { Principios básicos de adaptación } \\
\text { y evolución }\end{array}$ & & Producto Turístico & \multirow{9}{*}{$\begin{array}{l}\text { Módulo } \\
\text { II } \\
\text { Negocio } \\
\text { S } \\
\text { Producti } \\
\text { vos }\end{array}$} & Elección de la idea \\
\hline \multirow{8}{*}{$\begin{array}{l}\text { Módulo } \\
\text { II } \\
\text { Principio } \\
\text { s Básicos } \\
\text { Generale } \\
\text { s }\end{array}$} & Procesos de Especiación & & Perfiles del Turista & & $\begin{array}{l}\text { Planteamiento de } \\
\text { objetivos }\end{array}$ \\
\hline & Cadena Alimenticia & Módulo & Agencias de & & Determinación de \\
\hline & & II & Viajes & & funciones \\
\hline & Presiones humanas sobre el & Prestado & Alojamiento & & Desarrollo de la idea \\
\hline & medio natural & res de & Turístico & & \\
\hline & Procesos de extinción global & Servicio & Transportación & & Tiempo de ejecución \\
\hline & Cambio climático & $\begin{array}{l}\text { S } \\
\text { Turístico }\end{array}$ & $\begin{array}{l}\text { Turística } \\
\text { Entretenimiento }\end{array}$ & & Financiamiento \\
\hline & & $\mathrm{s}$ & & & \\
\hline
\end{tabular}

Fuente: Elaboración propia.

\section{Planificando los métodos}

La Andragogía requiere de métodos activos de aprendizaje, en los que quien aprende juega un papel activo, actúa, usa su potencial, busca, y obtiene por sí mismo/a resultados; en otras palabras "aprende haciendo". Con la planificación de los métodos, la propuesta metodológica busca lograr diversos objetivos tales como: facilitar la información, comprensión de conceptos, logro de un mejor autoconocimiento o conocimiento de otros, consolidación de equipos de trabajo, y el uso de herramientas y métodos para el manejo de problemas específicos.

La propuesta deja planteadas y desarrolladas cinco técnicas activas de aprendizaje que son: 
- Método socrático: donde el facilitador va a generar pregunta tras pregunta hasta encontrar la verdad o al menos una respuesta correcta; se genera una lluvia de preguntas para desencadenar un pensamiento crítico.

- Método por ensayo y error: donde los educandos prueban una opción y se identifica si es la correcta; si funciona, entonces se tiene una solución; si por el contrario no funciona, esto es un error; por lo que se debe intentar una y otra vez hasta llegar a lo correcto.

- Método de proyecto: se basa en un problema real, donde los educandos crean oportunidades de investigación para generar nuevos conceptos, aplicar la información, y representar el conocimiento frente al grupo para llegar a conclusiones generales.

- Método de casos: el facilitador expone una situación concreta para que los educandos la estudien, definan los problemas, lleguen a sus propias conclusiones sobre las acciones que habría que emprender, contrasten ideas, las defiendan y las reelaboren con nuevas aportaciones.

- Aprendizaje vivencial o experiencial con técnicas de outdoor adventure y outdoor training que buscan generar un conocimiento más fuerte desarrollando actividades al aire libre.

Con el desarrollo de estos métodos, el adulto según Requejo (2003) aprende a lo largo de toda su vida, se convierte en un estudiante activo, basando su proceso de aprendizaje en la acción.

\section{Aplicando el programa}

Esta fase del ciclo andragógico, permite convertir la experiencia en aprendizaje, al generar cambios comportamentales e ir más allá del encuentro educativo; este conocimiento para Requejo (2003) debe partir de las experiencias previas del alumno y volver de manera necesaria a su experiencia real, aprender desde el Constructivismo ${ }^{5}$. Una vez que se hayan aplicado las técnicas en cada uno de los módulos, las experiencias obtenidas por parte de los participantes deben transformarse en experiencias reales. El aprendizaje llega a ser significativo cuando se interioriza; por lo que se debe cumplir con el siguiente microciclo:

\footnotetext{
${ }^{5}$ El constructivismo es una corriente pedagógica basada en la teoría del conocimiento constructivista, que postula la necesidad de entregar al estudiante las herramientas necesarias (generar andamiajes) que le permitan construir sus propios procedimientos para resolver una situación problemática, lo que implica que sus ideas puedan verse modificadas y siga aprendiendo.
} 


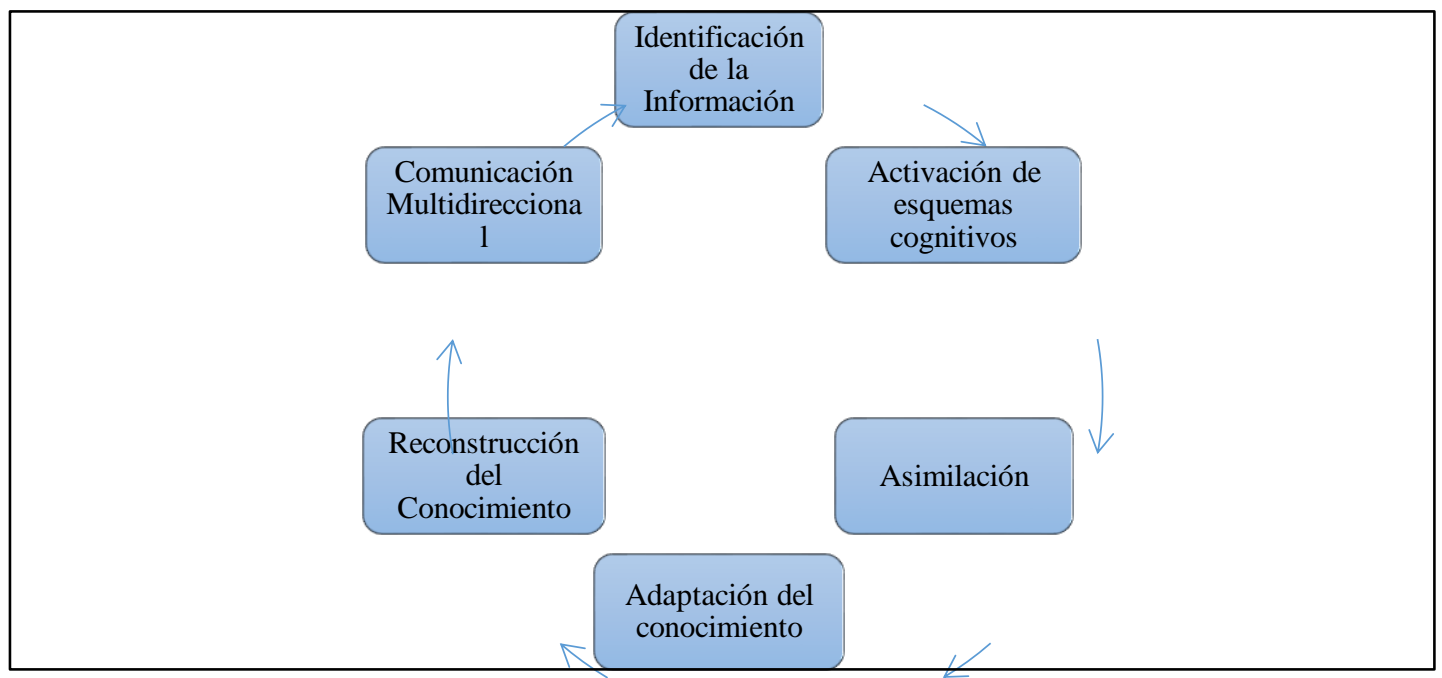

Figura 2 Ciclo de aplicación del programa andragógico. Según Agustín Requejo, en el libro Educación Permanente y Educación de Adultos, 2003.

El microciclo parte de la adquisición de un nuevo conocimiento, luego los educandos reconocen el aprendizaje previo para generar una sinergia entre ambos, para estar en la facultad de aplicar el conocimiento en la vida real, agregándole un sello original; de esta forma los educandos ya pueden compartir su conocimiento con familiares u otros emprendedores turísticos. Solo una vez que se haya cumplido con este microciclo, se puede hablar de una interiorización del conocimiento. Este proceso se debe cumplir en todas las asignaturas y con todos los métodos que se plantearon en el punto anterior; todos sin excepción alguna, contribuyen a que se cree un conocimiento perdurable en el tiempo en todos los participantes.

El presente Plan de Capacitación asegura el desarrollo de la actividad turístico ambiental dentro del Chocó Andino de Pichincha, tomando en cuenta las principales líneas de acción, elementos y realidades del turismo en la zona. El programa contempla un total de 284 horas de capacitación, divididas en 220 horas teóricas vivenciales y 64 horas de campo, en 26 módulos o asignaturas divididas en 3 grandes ejes; y pretende dotar de una verdadera visión turístico - ambiental a todos los participantes.

\section{Evaluando los resultados}

Los indicadores de evaluación, dentro de un proceso de capacitación local, si bien no tienen un papel determinante, ayudan a establecer el nivel de conocimientos alcanzados e interiorizados por parte de la comunidad. El objetivo del proceso evaluativo no puede estar dirigido sólo a una certificación de aprendizajes logrados o no logrados, sino que debe apuntar a establecer niveles de avance o progreso en el acercamiento a un determinado conocimiento, en consideración a una incorporación significativa acorde con los conocimientos previos que posee cada educando.

Por lo tanto, para concluir con el ciclo andragógico, se estableció un modelo de evaluación cuantitativo y cualitativo. Por una parte, incluye preguntas de respuesta simple, identificación de componentes, de ordenamiento, asociación de hechos, de verdadero y falso con 
un puntaje ponderado; y por otra, también ayuda a valorar el nivel de aprovechamiento alcanzado por los alumnos en el proceso de aprendizaje tomando en cuenta el principio de devolución. Generar una aprobación o no aprobación de los módulos es necesaria, en términos de medición de resultados para las entidades que financian los procesos de capacitación.

\section{Conclusiones}

La metodología de capacitación para emprendimientos turísticos del Chocó Andino de Pichincha, satisface la necesidad que tiene la comunidad de adquirir conocimientos técnico científicos qué complementados con la experiencia y conocimientos empíricos, buscan crear bases reales sobre cómo manejar la actividad turístico - ambiental en ecosistemas considerados como sensibles por su inmensa riqueza biológica y cultural.

Se define al modelo andragógico, como la mejor herramienta porque favorece al diálogo, origina puntos de vista que conducen a plantear propuestas y conclusiones grupales. El modelo andragógico adaptado a la capacitación turístico-ambiental implica crear un nuevo rol en el educando, donde el proceso de aprendizaje implique análisis de las situaciones, participación activa, compartir experiencias, generar soluciones y nuevos conocimientos a la comunidad. Los planes de capacitación en ecosistemas con una realidad socioeconómica vulnerable, no sólo deben ser aplicados, sino por el contrario implican establecer un plan de continuidad de aprendizaje, con el fin de actualizar los conocimientos que los participantes adquirieron; solo así lograrán afianzar e interrelacionar conocimientos con experiencia.

La capacitación turística ambiental debe ser un proceso planificado desde la propia realidad local, satisfacer la demanda participativa, estar basada en los recursos culturales, naturales y de conocimiento tradicional de la comunidad, y estar estructurada en torno a la habilidad creativa de los participantes. La Andragogía, de esta forma, contribuye a la revitalización de saberes, refuerza la autoestima de los participantes y favorece el aprendizaje cooperativo. Un proceso de capacitación integral es fundamental para que la comunidad valore la riqueza cultural y ambiental del Chocó Andino de Pichincha, de esta forma se pueden establecer políticas locales en temas de ambiente y turismo que por una parte posicionen el producto turístico a nivel regional, y por otra, conserven la riqueza y la protejan de riesgos ambientales.

El principal objetivo de un proceso de capacitación participativo es construir una industria turística sostenible con empoderamiento local que se aleje del paradigma construido desde el norte global. Un nuevo paradigma donde conservación y sostenibilidad sean categorías construidas desde lo local, una construcción desde abajo, desde el empoderamiento comunitario poseedor de una cultura propia y una diversidad biológica que son los medios para insertarse en el mercado global. Se crea, de esta manera, una construcción de lo local redefiniendo las estructuras de poder, las instituciones, las competencias para que generen viabilidad económica dentro de las estructuras productivas locales. 


\section{Bibliografía}

Aguilar, D. (2014). Proyecto para la creación de una escuela de capacitación de mandos medios para el sector hotelero de la ciudad de Quito. Quito, Ecuador: Tesis de pregrado Universidad Politècnica Salesiana.

Benne, K. (2005). De la Pedagogía a la Antropogogía. Madrid, España: Ediciones Rialp.

Caiza, P. (2017). Sustentabilidad y el Estado constitucional de derechos intercultural y plurinacional - ecuatoriano. Quito, Ecuador: Flacso.

Caiza, P. (2018). Ensayo El turismo comunitario, una alternativa al desarrollo local desde la cooperación internacional. Quito, Ecuador: Flacso .

Caiza, P., \& Peralta, L. (2017). Turismo sostenible, desde un enfoque de sustentabilidad fuerte: Casos de estudio Yunguilla y Mindo. Quito, Ecuador: Flacso.

Carr, D. (2005). El sentido de la Educación. Una introducción a la filosofía y a la teoría de la educación y de la enseñanza. Barcelona, España: Grao Editorial.

Castiblanco Rozo, C. (2015). Los paradigmas de la sostenibilidad. Bogotá: Cepal. Recuperado el 16 de abril de 2019, de https://www.cepal.org/sites/default/files/.../presentacion_carmenza_castiblanco.pdf

Catalano, A., Avolio de Cols, S., Sladogna, M. (2004). Diseño curricular basado en normas de competencia laboral: conceptos y orientaciones metodológicas. Buenos Aires, Argentina: Banco Interamericano de Desarrollo.

Dewey, J. (1938). Experience and Education. New York, USA: Macmillan Company.

Gallegos, M. (2009). El desarrollo humano sustentable no es posible en el capitalismo. La contrucción de algunas alternativas desde abajo. Herramienta Revista de debate y crítica marxista, 28.

Gobierno Provincial de Pichincha. (2015). Plan de Desarrollo y Ordenamiento Territorial Pichincha 2015-2019. Quito, Ecuador: Consejo Provincial de Pichincha.

Gudynas, E. (2009). Desarrollo Sostenible: posturas contemporáneasy desafíos en la construcción del espacio urbano. Vivienda Popular, 18, 12-19.

Leff, E. (2000). Globalización, ambiente y sustentabilidad del desarrollo. En E. Leff, Saber ambiental: sustentabilidad, racionalidad, complejidad, poder (págs. 17-30). México DF, México: Siglo XXI.

Ludojoski, R. (1978). Antropogogía o la Educación del Hombre: introducción al problema de la Antropología educativa en la perspectiva de la educación permanente. Barcelona, España: Editorial Guadalupe.

Martínez Alier, J., Roca Jusmet, J. (2013). El Debate de la Sustentabilidad. En J. Martínez Almier, J. Rooca Jusmet, Economía ecológica y política ambiental (págs. 459-518). México DF, México: Fondo de Cultura Económica.

McClelland, D.C. (1973). Testing for Competence Rather than for Intelligence. American Psychologist, 28, 1-14.

Meadows, D. (1983). Los Límites del crecimiento. En A. Dowson, Pensamiento verde: una antología (págs. 21-25). Madrid, España: Trotta.

Nonaka, I., Toyoma, R., Konno, N. (2000). SECI, Ba and leadership: a unified model of dynamic knowledge creation. Long range planning, 33(1), 5-34.

Organización Naciones Unidas. (1972). Informe de la Conferencia de las Naciones Unidas sobre el Medio Ambiente. Nueva York, USA: ONU. 
Organización Naciones Unidas. (1987). Informe de la Comisión Mundial sobre el Medio Ambiente y el Desarrollo, Nuestro Futuro Común. Nairobi, Kenia: ONU.

Otero, A. (2006). La formación de recursos humanos en turismo y recreación para la competitividad regional de la Patagonia Argentino Chilena. Aportes y Transferencias, 10 (1), 62-77.

Pastor Alonso, M., Espeso Molinero, P. (2015). Capacitación turística en comunidades indígenas. Un caso de Investigación Acción Participativa (IAP). El Periplo Sustentable, (29), 171208.

Pérez, G., Pineda, U., \& Arango, M. (2011). La capacitación a través de algunas teorías de aprendizaje y su influencia en la gestión de la empresa. Revista Virtual Universidad Católica del Norte(33), 1-22.

Requejo, A. (2003). Educación Permanente y Educación de adultos. Barcelona, España: Editorial Ariel.

Rodríguez Rojas, P. (2003). La andragogía y el constructivismo en la sociedad del conocimiento. Laurus, 9(15), 80-89.

Shalk Quintana, A. E. (2009). Modelo de Enseñanza - Aprendizaje para Adultos en la era del conocimiento. Diseño y Estructura del Modelo. Barcelona, España.

Torres, M., Fermín, Y., Arroyo, C., \& Piñero, M. (2000). La horizontalidad y la participación en la Andragogía. Educere, 4(10), 25-34.

Zalles, J. (2016). El gallito de la peña: turismo, uso de suelo y conservación biológica en el noroccidente de Pichincha, Ecuador. Quito, Ecuador: Flacso. 